Douleur Neuropathique 4 (DN4) stratifies possible and definite neuropathic pain after surgical peripheral nerve lesion

\title{
Aho, Tommi
}

2020-02

Aho , T , Mustonen , L , Kalso , E \& Harno , H 2020 , ' Douleur Neuropathique 4 (DN4) stratifies possible and definite neuropathic pain after surgical peripheral nerve lesion ' European Journal of Pain , vol. 24 , no. 2 , pp. 413-422 . https://doi.org/10.1002/ejp.1498

http://hdl.handle.net/10138/327208

https://doi.org/10.1002/ejp.1498

acceptedVersion

Downloaded from Helda, University of Helsinki institutional repository.

This is an electronic reprint of the original article.

This reprint may differ from the original in pagination and typographic detail.

Please cite the original version. 
Article Type: Original Manuscript

\section{Douleur Neuropathique 4 (DN4) stratifies possible and definite neuropathic pain after surgical peripheral nerve lesion}

Running head: DN4 stratifies possible and definite neuropathic pain.

T. Aho ${ }^{1}$, L. Mustonen ${ }^{1,2}$, E. Kalso ${ }^{1}$ and H. Harno ${ }^{1,2}$

1Division of Pain Medicine, Department of Anaesthesiology, Intensive Care and Pain

Medicine, University of Helsinki and Helsinki University Hospital, Finland

${ }^{2}$ Neurocenter, Neurology, University of Helsinki and Helsinki University Hospital,

Finland

\section{Corresponding author:}

Tommi Aho

Pain Clinic

Helsinki University Hospital

Haartmaninkatu $2 \mathrm{~A}$

P.O. Box 140

00029 HUS Helsinki, Finland

Tel. + 358504639469

e-mail: tommi.aho@fimnet.fi

Submission Category: Original article

This article has been accepted for publication and undergone full peer review but has not been through the copyediting, typesetting, pagination and proofreading process, which may lead to differences between this version and the Version of Record. Please cite this article as doi: 10.1002/EJP.1498

This article is protected by copyright. All rights reserved 
Funding source: This research was founded by the European Union FP7 (\# Health_F2-2013-602891).

Conflicts of interest: The authors state that there are no conflicts of interest.

Significance: DN4 stratifies possible and definite postsurgical peripheral neuropathic pain. DN4i may also show this, but full DN4 is more accurate. We confirm DN4i as a valid screening tool for NP.

\section{ABSTRACT}

Backgroung: Douleur Neuropathique 4 (DN4) is a screening questionnaire to help identify neuropathic pain (NP) in clinical practice and research. We tested the accuracy of the DN4 questionnaire in stratifying possible NP (pNP) and definite NP $(\mathrm{dNP})$ in patients operated for breast cancer.

Methods: We studied 163 patients from a longitudinal cohort of breast cancer operated patients 4-9 years after surgery. pNP or dNP were classified according to the NP grading system. Surgeon-verified intercostobrachial nerve resection was used as a confirmatory test for dNP. A receiver-operating characteristic $(R O C)$ curve analysis was performed and the area under the curve (AUC) was calculated to test the diagnostic accuracy (sensitivity, specificity, positive and negative predictive values) of the DN4. Additionally, we studied clinical factors that associated with a positive screening outcome in the interview part of the DN4 (DN4i).

Results: DN4i and DN4 showed significant accuracy in stratifying patients with pNP or dNP with cut-off scores 3 and 4 resulting to sensitivity of $66.2 \%$ and $79.4 \%$ and specificity of $77.8 \%$ and $92.6 \%$, respectively. pNP and dNP patients showed 
differences in sensory descriptors of pain according to DN4i items. Screening positive on DN4i associated with dNP and younger age.

Conclusions: Full DN4 could stratify pNP and dNP patients in a chronic postsurgical NP patient group operated for breast cancer. Additionally, DN4i showed significant accuracy in stratifying pNP and dNP, but an examination is necessary to obtain proper accuracy. Demographic factors may have an impact on the screening outcome of DN4i.

Keywords: Neuropathic pain, DN4, breast cancer, peripheral nerve lesion, postsurgical pain

\section{INTRODUCTION}

The clinical diagnosis of neuropathic pain (NP) is based on a lesion or disease of the somatosensory nervous system with pain and sensory abnormality in the neuroanatomically relevant area (Jensen et al., 2011). According to the revised NP diagnostic criteria (Finnerup et al., 2016), a surgeon-defined nerve lesion can be used to confirm definite NP (dNP).

In cases with surgeon-defined nerve resection, pain without sensory abnormalities on a neuroanatomically plausible area is regarded as possible NP (pNP) (Finnerup et al., 2016) and with sensory abnormalities, as definitely neuropathic (dNP).

Various screening tools have been developed to identify the main symptoms of NP and to help identify NP components (Attal et al., 2018; Bouhassira et al., 2008). NP is a common complication after breast cancer surgery and frequently associated with 
intercostobrachial nerve (ICBN) resection (Pereira et al., 2017; Mustonen et al., 2019). Screening tools may help identify postsurgical NP especially in conditions where postsurgical nerve lesion is difficult to identify (Attal et al., 2018). Additionally, screening tools for NP may provide information of the pathophysiological mechanisms associating with specific NP conditions and sensory phenotypes (Baron et al., 2012).

The Douleur Neuropathique 4 (DN4) is a screening questionnaire developed to assess both sensory descriptors and signs that relate to NP bedside sensory examination (Bouhassira et al., 2005). DN4 has been widely used in various NP states with different etiologies (Bouhassira et al., 2005; Mathieson et al., 2015; VanDenKerkhof et al., 2018; Attal et al., 2018).

The last three questions in DN4 relate to the bedside sensory examination. These have been suggested to be performed as the first step screening examinations for NP in routine clinical practice to evaluate the need for further confirmatory diagnostic tests or neurological examination (Attal et al., 2018). In the interview part of the DN4 (DN4i), NP- related sensory descriptors may have relevance in general epidemiology of neuropathic characteristics of pain in chronic pain patients (Bouhassira et al., 2008).

Previously, a positive correlation between DN4 and probable NP has been shown with surgical nerve lesions, such as in post-thoracotomy pain (Guastella et al., 2011), pain after lymph node excision (Høimyr et al., 2011), and pain after breast tumor resection (Abdallah et al., 2015). However, it is not known whether DN4 is sensitive to stratify pNP and dNP. To address this question, we studied DN4 and DN4i in parallel in patients with pNP and dNP from a cohort of patients previously operated for breast cancer with a surgeon-defined resection of the ICBN (Mustonen et al., 2019). Additionally, as sensitivity of the questionnaire may be influenced by clinical and demographic characteristics (VanDenKerkhof et al., 2018), we studied factors that associated with a positive screening outcome of the DN4i. 


\section{METHODS}

\section{Patients and neuropathic pain assessment}

The patients of the present study were recruited from an earlier longitudinal study cohort of 1000 women treated for breast cancer (Kaunisto et al., 2013). 251 patients with surgeon-verified total or partial ICBN resection were recruited for the current cohort. Patient recruitment has previously been described in more detail elsewhere (Mustonen et al., 2019). All patients underwent either breast conserving surgery (BCS) or mastectomy in combination with either sentinel lymph node biopsy (SLNB) or axillary lymph node dissection (ALND). We have previously assessed factors that related to persistent postsurgical NP 4 to 9 years after surgery in patients with ICBN lesion (Mustonen et al., 2019). There, we examined the patients at a research visit and used the NP grading system (Finnerup et al., 2016) to identify pNP and dNP. The study was conducted at the Pain Clinic of the Helsinki University Hospital, Helsinki, Finland during 2014-2016. The study protocol was approved by the Ethics Committee of the Helsinki and Uusimaa Hospital District. All patients gave an informed written consent.

We used the revised stepwise NP grading system for patient classification (Finnerup et al., 2016, Mustonen et al., 2019). The steps include the following: A) pain in the neuroanatomically plausible area with relevant pain history, B) pain associated with at least one sensory abnormality in clinical examination, and $C$ ) surgeon-verified nerve lesion. After breast cancer surgery, the neuroanatomically plausible area includes the breast and the ICBN innervation area (axilla, upper side of chest, lateral breast and medial upper arm) (Andersen et al., 2014). pNP patients had pain in the neuroanatomically plausible area, but without signs of sensory abnormalities (only step $A$ fulfilled). dNP patients had pain with sensory abnormalities within the innervation area of the resected ICBN (steps A, B and C fulfilled) (Fig. 1). 
All patients in this cohort had a surgeon-verified ICBN resection and this served as a confirmatory test (step $\mathrm{C}$ ) to reach the diagnostic level of dNP. The patients with pain and sensory changes only outside the ICBN innervation area (e.g. in medial breast) were excluded. In addition, patients without pain (no self-reported or evoked pain) and patients with ongoing cancer treatments were excluded from the analyses.

A neurologist $(\mathrm{HH})$ examined the clinical sensory status as follows: static mechanical allodynia (compression by finger), light touch (cotton tuft), dynamic touch (painter's brush), pinprick (wooden cocktail stick), cold and warm sensation (metal roller). The metal roller was dipped into ice cold water bucket (cold water with ice cubes in it) before applying sensory examination for cold. Respectively, the metal roller was dipped into hot water before applying sensory examination for warm sensation. Hot water was in an electric water boiler and the water had been boiled just before dipping the metal roller there. No specific temperature measurement was performed. The examiner tested the roller's temperature to her own skin before testing the patient to ensure tolerability of the testing temperature. The sensory examination needed for DN4 was included in the clinical sensory status. DN4i was performed by reading the questions to the patient out loud.

The examiner was blinded to the ICBN status (no lesion, partial, total, or not visualized ICBN lesion in surgeon report) and after all patients had been examined, the code was opened for NP classification and the ones with ICBN lesion and pain accordingly were chosen for the present study. For illustration, we used patient pain drawings and body maps for sensory examination performance. We combined these data with the ICBN status and could classify patients according to NP grading system (Mustonen et al., 2019).

\section{Douleur Neuropathique 4 (DN4)}

The DN4 questionnaire was administered at the research visit by a single researcher $(\mathrm{HH})$ to assess neuropathic components of pain (Bouhassira et al., 2005). The DN4 consists of ten items with a dichotomous yes-no scale. The first part of the 
questionnaire (items 1 to 7, i.e. interview part, DN4i) consists of seven self-report items about pain descriptors: 'Does the pain have one or more of the following characteristics? - 1) burning, 2) painful cold, 3) electric shocks' and 'Is the pain associated with one or more of the following symptoms in the same area? -4) tingling, 5) pins and needles, 6) numbness, 7) itching'. The second part of the questionnaire (items 8 to 10, i.e. examination part) consist of three items relating to sensory examination: 'Is the pain located in an area where the physical examination may reveal one or more of the following characteristics? - 8) hypoesthesia to touch, 9) hypoesthesia to pinprick' and 10) 'In the painful area, can the pain be caused by brushing?'. Thus, the scores range from 0-7 and 0-10 for DN4i and DN4, respectively.

We considered scores $\geq 3$ for DN4i and $\geq 4$ for DN4 as cut-offs for suggestive NP, i.e. a positive screening outcome (Bouhassira et al., 2005). At the research visit, the interview part (DN4i) was fulfilled and the examining neurologist completed the three-item clinical part of the DN4. From here on we use the abbreviation DN4i for the DN4 interview part and DN4 for the full DN4.

\section{Demographics, questionnaires and pain assessment}

Demographics were collected at the research visit by multiple questionnaires. We used the following variables for psychological factors: Hospital Anxiety and Depression Scale (HADS) for assessing anxiety and depressive symptoms (Bjelland et al., 2002) and Pain Catastrophizing Scale (PCS) for assessing pain associated catastrophic thinking (Sullivan et al., 1995).

For pain intensity, we used the worst pain during past week in the operated area measured on a Numerical Rating Scale (NRS from 0 to 10, 0 indicating no pain and 10 the worst imaginable pain intensity) of the Brief Pain Inventory (BPI) (Cleeland et al., 1994). At clinical examination, possible evoked pain was rated by NRS from 0 to 10 , respectively. Patients reported worst other pains (apart from those in the 
operated area) with BPI during past week (NRS 0-10). We considered NRS $\geq 4 / 10$ as moderate to severe pain.

\section{Statistical analysis}

We used Student's t-tests, Mann-Whitney U-tests and $\mathrm{X}^{2}$-tests for continuous normally and non-normally distributed variables and categorical variables, respectively. Spearman's rho $\left(r_{S}\right)$ was used for correlation of continuous predictor variables. Cronbach's alpha was used for assessing internal consistency of the questionnaires. Logistic regression analysis was performed to explore factors, which may associate with positive screening in DN4i (sum score $\geq 3$ ). Predictors for logistic regression were selected based on correlations with sum scores of DN4i. We used backward stepwise method to control for multicollinearity of anxiety (HADS-A) and depression (HADS-D). For the same reason, as pain variables in the operated area showed strong correlation, only intensity of worst pain in the operated area was entered to the final model. Calibration of the logistic model was tested with HosmerLemeshow test ( $p>0.05$ was preferred).

For analyzing stratifying accuracy of the DN4 questionnaire, we calculated sensitivity, specificity, and positive and negative predictive values with $95 \%$ confidence intervals. We used sum score of 3 and 4 as a cut-off values for NP in DN4i and DN4, respectively (Bouhassira et al., 2005). However, Youden index was also used to confirm the optimal cut-off values in our data. As the original version of DN4 has been validated in patients with at least moderate worst pain intensity, we additionally run the analyses separately for patients reporting at least moderate worst pain (NRS $\geq 4 / 10$ ) in the operated area. A receiver-operating characteristic (ROC) curve analysis was performed and the area under the curve (AUC) was calculated. P-values under 0.05 were considered statistically significant. We used SPSS 22.0 version for Windows (SPSS Inc., Chicago, IL., USA). 


\section{RESULTS}

\section{Patients and demographics}

The patient flow in the present study is illustrated in Fig. 1. Altogether, 27 pNP and 136 dNP patients were included in the analyses.

Demographics and clinical features of the patients are presented in Table 1. dNP patients were significantly younger than pNP patients $(p=0.007)$. Moderate to severe evoked pain in the postsurgical or nearby area was the most common pain type for both pNP and dNP.

Patients with dNP reported more often pain elsewhere in the body (NRS $\geq 4)$ $(p=0.044)$ and had received chemotherapy more often than patients with $p N P$ $(p=0.039)$.

In both groups, the use of NP medications was scarce. Patients did not use analgetics regularly and did not use strong opioids.

In clinical sensory examination of the painful postsurgical or nearby area, dNP patients presented commonly with hypoesthesia for cold and warm, in 134 patients (98.5\%) and 132 (97.1\%), respectively. Additionally, for cold and warm, they presented with hyperesthesia 12 (8.8\%) and 11 (8.1\%), dysesthesia $15(11 \%)$ and 2 $(1.5 \%)$, and allodynia $3(2.2 \%)$ and 0 patients $(0 \%)$, respectively. 


\section{Sensory descriptors of the DN4i and DN4}

In Fig. 2, we present the sensory descriptors of DN4i and clinical sensory findings in the full form of DN4. Of the sensory descriptors, dNP patients reported more often numbness $(p<0.001)$, pins and needles $(p<0.001)$, tingling $(p=0.020)$, and electric shocks $(p=0.032)$ compared with pNP patients. We found no differences between pNP and dNP on itching, painful cold, nor on burning.

Of the clinical sensory findings in full DN4, $11 \mathrm{dNP}$ patients $(8.1 \%)$ did not show those in full DN4, but other sensory abnormalities. Most of the dNP patients $(72.8 \%$, 99/136) showed abnormalities in two DN4 sensory findings, whereas $16.2 \%(22 / 136)$ showed abnormalities in one item, and $2.9 \%(4 / 136)$ in all three items. The most common clinical sensory finding was hypoesthesia to touch $(84.6 \%, 115 / 136)$, following hypoesthesia to pinprick in $75.5 \%$ (103/136), and painful brushing in $10.3 \%$ (14/136). Patients with pNP did not present any clinical sensory findings in the plausible painful area (Fig. 2).

\section{Screening outcome of the DN4i}

The sum score of DN4i showed significant negative correlation with age $(p<0.001)$, but positive correlation with other variables: intensity of self-reported and evoked pain $(p<0.05)$, intensity of worst pain in the operated area $(p<0.05)$, intensity of other chronic pains $(p<0.001)$, anxiety $(p<0.05)$, depression symptoms $(p<0.001)$, and pain catastrophizing ( $p<0.01)$ (Table 2). Intercorrelations between age, psychological, and pain variables are presented in the Table S1.

In logistic regression analysis, age showed significant association over all other correlating variables (adjusted OR 0.88, 95\% Cl: 0.83-0.94), $\mathrm{p}<0.001$ ) with a positive DN4i screening (i.e. sum score $\geq 3$ ) when the dNP was controlled (Table S2).

\section{Stratifying accuracy of the DN4i and DN4}


DN4i presented $66.2 \%$ sensitivity and $77.8 \%$ specificity (Table 3). Of the patients, $68.1 \%(111 / 163)$ were correctly identified (highest Youden index value of 0.44 ,Table S3). The DN4i sum score presented $\geq 3$ in $96 / 163$ patients (58.9\%). pNP and dNP was significantly stratified by DN4i (AUC=0.762, 95\% Cl: 0.661-0.863, $p<0.001)$ (Fig. 3).

Full DN4 presented $79.4 \%$ sensitivity and $92.6 \%$ specificity (Table 3 ). Of the patients, $81.6 \%$ (133/163) were correctly identified (highest Youden index value of 0.72 , Table S3). The DN4 sum score presented $\geq 4$ in $110 / 163$ patients $(67.5 \%)$. Two patients with pNP screened a sum score $\geq 4$ on DN4. pNP and dNP were significantly stratified by DN4 (AUC=0.865, 95\% Cl: 0.768-0.962, $p<0.001$ ) (Fig. 3).

We analyzed separately the DN4i and DN4 sensitivity and specificity for the patients with moderate to severe worst pain intensity (NRS $\geq 4, n=113$ ) in the postsurgical or nearby area. Here, the sensitivity of DN4i was $67.7 \%$ and specificity $76.5 \%$ whereas the sensitivity of DN4 was $79.2 \%$ and specificity $94.1 \%$ (Table 3). Of the patients, $69.0 \%$ (78/113) and $81.4 \%$ (92/113) were correctly identified, respectively. pNP and dNP were significantly stratified by both DN4i (AUC $=0.770,95 \% \mathrm{Cl}: 0.646-0.894$, $p<0.001)$ and DN4 (AUC=0.881, 95\% Cl: 0.768-0.969, $p<0.001)$.

\section{DISCUSSION AND CONCLUSIONS}

DN4 showed significant accuracy in stratifying pNP and dNP after surgical peripheral nerve lesion in breast cancer operated patients. DN4i showed significant accuracy as well, but clinical sensory examination is needed for proper NP diagnostics. Sensory descriptors of pain in the DN4i items differed between pNP and dNP patients. dNP patients reported more often numbness, pins and needles, tingling, and electric shocks compared with pNP. Positive screening in DN4i showed significant correlation between clinical and psychological variables and associated with dNP and younger age. 
It is challenging to verify dNP in postsurgical patients (Abdallah et al., 2015; Guastella et al., 2011; Høimyr et al., 2011). NP questionnaires are needed to help stratify NP patients. To the best of our knowledge, this is the first study to show that with DN4 one may stratify chronic postsurgical NP patients to pNP and dNP groups and that DN4i shows significant accuracy as well. However, only clinical sensory examination provides proper diagnostic accuracy for NP.

DN4 was designed as a screening tool for NP, something to be used first line by non-specialists (Bouhassira et al., 2005). In previous studies, DN4i and DN4 have been sensitive and specific enough to help in NP diagnostics (Bouhassira et al., 2005; Hamdan et al., 2014; Chatila et al., 2017; Timmerman et al., 2017). In our cohort, we found that DN4i may be useful as a first step screening of NP in patients with surgical nerve resection.

Sensitivity and specificity are key elements in evaluating DN4 validity in stratifying pNP and dNP. A previous study used DN4 to screen postsurgical NP in breast cancer treated patients. They reported DN4 sensitivity and specificity to be $90 \%$ and $60 \%$, respectively (Abdallah et al., 2015). In line with these, our results of full DN4 sensitivity and specificity were $79.4 \%$ and $92.6 \%$, respectively. Previously, DN4 has correlated to pain severity (Perez et al., 2007). Thus, we analysed sensitivity and specificity in patients with pain intensity $\geq 4$ on a numerical rating scale (NRS). Now, DN4 sensitivity and specificity were $79.2 \%$ and $94.1 \%$, respectively. These results encourage using DN4 in clinical practice to screen and even stratify pNP and dNP.

Previously, NP has been screened by DN4i in chronic pain patients, including breast cancer treated patients (Timmerman et al., 2017). The authors reported DN4i sensitivity and specificity of $70 \%$ and $67 \%$, respectively. In line with these, sensitivity and specificity for DN4i in our cohort was $67.7 \%$ and $76.5 \%$, respectively. DN4i may be a feasible tool in screening NP in large cohorts (Bouhassira et al., 2005).

It is important to report positive and negative predictive values of a studied questionnaire. In a previous study of screening NP in chronic pain patients, DN4 
showed positive and negative predictive values of $92 \%$ and $46 \%$, respectively (Timmerman et al., 2017). In line with these, our data for DN4i and full DN4 showed positive predictive values of $93.8 \%$ and $98.2 \%$ and negative predictive values of $31.3 \%$ and $47.2 \%$, respectively. These results implicate that if DN4 provides a negative result, it might be false negative, i.e. the patient may anyhow have NP. This is especially important to consider if DN4 is used in clinical practice.

Patients with NP may have other sensory abnormalities and pain features than what are studied by DN4. In our cohort, $8.1 \%$ of dNP patients had additional other sensory abnormality (such as hyperesthesia and/or changes in thermal sensation) that are not included in the DN4. This is important to consider especially in cases of negative screening.

Sensory loss to both mechanical and thermal stimuli is common in patients with chronic postsurgical pain after breast cancer surgery (Andersen et al., 2017; Mustonen et al., 2019), but also hypersensitivity has been reported (Gottrup et al., 2000; Vilholm et al., 2009). Tingling and numbness, but also other DN4 pain characteristics, were common reports in a previous study on chronic pain patients including also breast cancer operated women. In the clinical examination, hypoesthesia to touch was the most prevalent (Timmerman et al., 2017). In our DN4 data, patients with dNP reported and presented most typically hypoesthesia to touch and pinprick. These findings are in line with other reports of sensory abnormalities after peripheral nerve lesion (Gottrup et al., 2000; Vilholm et al., 2009; Abdallah et al., 2015; Andersen et al., 2017; VanDenKerkhof et al., 2018).

dNP and pNP patients did not differ in terms of burning pain and itching. Itching involves complex mechanisms both at the peripheral and spinal level, which may be influenced by the existing neuropathy (Steinhoff et al., 2019). In a large population survey, itching was associated with chronic pain (Dalgard et al., 2007). We are not aware of any studies on the role of itch in chronic post-surgical pain. Our results are in line with a previous study on DN4 in chronic pain patients where itching did not associate with NP component (Timmerman et al., 2017). 
In a study of diabetic patients, burning pain was associated with epidermal nerve regeneration (Galosi et al., 2018). Our results with relatively infrequent reports on burning pain may indicate that other mechanisms are more prevalent in the pathophysiology of post-surgical NP after breast cancer treatments.

Previously, psychological variables, such as catastrophizing and mood, have shown to have an impact on the screening outcome of the full DN4 questionnaire (VanDenKerkhof et al., 2018). Emotional functions and psychological distress, have a well-known impact on the severity of NP and may increase disability (Jensen et al., 2007; Bostic et al., 2017; Mustonen et al., 2019). In our cohort, sum scores of DN4i correlated significantly with age, pain variables, and psychological variables. However, only dNP and younger age associated with positive screening in DN4i.

Most of our patients presented evoked pain with little or no self-reported pain. Evoked pain does not necessarily correspond to clinically meaningful pain but may cause the patient to adapt to certain everyday-life habits to prevent pain. Recently, evoked pain has more systemically been acknowledged and can be classified as NP even in the absence of self-reported pain (Arning and Baron, 2011; Themistocleous et al., 2016; Mustonen et al., 2019).

The pathophysiological mechanisms of pain in pNP, where no sensory abnormality presents, remain diverse. Pain may be related to breast cancer or its treatments such as nerve injury, axon degeneration, infection, hormonal therapy, chemotherapy, or radiotherapy. Later, peripheral and central sensitization takes place with changes in neuroplasticity in the periphery, dorsal horn, and central nervous system (von Hehn et al., 2012).

Previously, painful hypoesthesia has been shown to associate with loss of small and large fiber function (Baron et al., 2017). For NP after a surgical peripheral nerve lesion, there most probably is one aetiology, followed by other aetiologies to maintain 
the condition. These factors are e.g central sensitization and partial nociceptive deafferentation (Baumgärtner et al., 2002). Also, smaller peripheral nerve lesions may take place in surgery and add to NP aetiology. For breast cancer surgery, not only ICBN resection, but smaller nerve resections may contribute to NP.

An important issue is how to improve the usage of patient-reported information. DN4i may help identify e.g. painful hypoesthesia after surgical nerve lesion and lead to better NP diagnostics and treatment.

A strength of the study is a fairly large cohort of chronic postsurgical pain patients with surgeon defined ICBN resection. Patients were carefully diagnosed according to the NP grading system and could be clinically stratified to PNP and dNP groups. This enabled us to test the sensitivity and specificity of DN4 in this cohort.

There are several limitations in this study. The current patient cohort consists of patients treated for breast cancer and therefore the generalizability of the findings is limited. dNP patients had had chemotherapy more often compared with pNP patients. The impact of this remains unclear, because chemotherapy did not show significant difference between painful NP and painless ICBN lesion patients in a previous study of which the present one is a sub cohort of (Mustonen et al., 2019).

Thermal examination in the present study did not include exact temperature measurement, which is a limitation regarding the information of thermal sensitivity. However, the thermal testing procedure was similar for all patients.

The negative predictive value of full DN4 of $47 \%$ is a limitation. Scoring negative in DN4 may be a false negative result, which is important to consider in clinical practice.

False negative and positive cases pose an important clinical problem. However, sensitivity and specificity were good enough to use DN4 as a screening instrument. 
Patients with a negative (false or true) score, however, should be studied further with sensory examination.

The aetiology of NP may vary. There might be other reasons for chronic pain than chronic postsurgical NP, e.g. myofascial, inflammatory, or central sensitization. These reasons cannot be distinguished from dNP by the DN4.

Pain medications may impact the results in DN4i and DN4. However, in our cohort, the patients used NP medications scarcely. Thus, bias due to NP medication is not very likely.

We performed a logistic regression analysis to study clinical factors that associate with a positive screening outcome in DN4i. Subgroup analyses of these factors would have been a better approach. However, our sample size was too small for this, but large enough to show the association in logistic regression analysis, where age showed significant association over all other correlating variables.

This study shows that DN4 and possibly DN4i may help stratify dNP and pNP patients after peripheral nerve lesion (ICBN). A wider use of DN4 in the clinic might help the first-line physician to consider NP and try NP medication or to refer the patient to a pain specialist.

The sensory descriptors of DN4i were distinct in dNP and pNP, which may reflect NP pathophysiology related with surgical nerve injury.

\section{ACKNOWLEDGEMENTS}

We want to thank research nurse Eija Ruoppa for her excellent work. We are grateful to all patients who participated in the study. 


\section{AUTHOR CONTRIBUTIONS}

$\mathrm{EK}$ and $\mathrm{HH}$ contributed to the study design and data collection. $\mathrm{HH}$ conducted the clinical examination. TA and LM contributed to the data analyses and the making of the figures and tables. TA, LM, EK and HH contributed to the writing of the manuscript. All authors were involved in data interpretation, review and approval of the final version of the manuscript. 


\section{REFERENCES}

Arning K., Baron R. (2009). Evaluation of symptom heterogeneity in neuropathic pain using assessments of sensory functions. Neurotherapeutics, 6,738-48.

Abdallah, F.W., Morgan, P.J., Cil, T., Escallon, J.M., Semple, J.L., \& Chan, V.W. (2015). Comparing the DN4 tool with the IASP grading system for chronic neuropathic pain screening after breast tumor resection with and without paravertebral blocks: a prospective 6-month validation study. Pain, 156, 740-49.

Andersen, K.G., Duriaud, H.M., Kehlet, H., Aasvang, E.K. (2017). The Relationship Between Sensory Loss and Persistent Pain 1 Year After Breast Cancer Surgery. Journal of Pain, 18, 1129-1238.

Attal, N., Bouhassira, D., \& Baron, R. (2018). Diagnosis and assessment of neuropathic pain through questionnaires. The Lancet Neurology, 17, 456-66.

Baron, R., Förster, M., \& Binder, A. (2012). Subgrouping of patients with neuropathic pain according to pain-related sensory abnormalities: a first step to a stratified treatment approach. The Lancet Neurology, 11, 999-1005.

Baron, R., Maier, C., Attal, N., Binder, A., Bouhassira, D., Cruccu, G., ... \& Jensen, T. S. (2017). Peripheral neuropathic pain: a mechanism-related organizing principle based on sensory profiles. Pain, 158, 261-272.

Baumgärtner, U., Magerl, W., Klein, T., Hopf, H.C., \& Treede, R.D. (2002). Neurogenic hyperalgesia versus painful hypoalgesia: two distinct mechanisms of neuropathic pain. Pain, 96, 141-151. 
Bjelland, I., Dahl, A.A., Haug, T.T., Neckelmann, D. (2002). The validity of the Hospital Anxiety and Depression Scale. An updated literature review. J Psychosom Res, 52, 69-77.

Bostick, G.P., Kamper, S.J., Haanstra, T.M., Dick, B.D., Stitt, L.W., Morley-Forster, P., ... \& Smyth, C. (2017). Pain expectations in neuropathic pain: Is it best to be optimistic? European J Pain, 21, 605-613.

Bouhassira, D., Attal, N., Alchaar, H., Boureau, F., Brochet, B., Bruxelle, J., ... \& Vicaut, E. (2005). Comparison of pain syndromes associated with nervous or somatic lesions and development of a new neuropathic pain diagnostic questionnaire (DN4). Pain, 114, 29-36.

Bouhassira, D., Lantéri-Minet, M., Attal, N., Laurent, B., \& Touboul, C. (2008).

Prevalence of chronic pain with neuropathic characteristics in the general population. Pain, 136, 380-387.

Chatila, N., Pereira, B., Maarrawi, J., \& Dallel, R. (2017). Validation of a new Arabic version of the neuropathic pain diagnostic questionnaire (DN4). Pain Practice, 17, 78-87.

Cleeland, C.S. \& Ryan, K.M. (1994). Pain assessment: global use of the Brief Pain Inventory. Ann Acad Med Singapore, 23, 129-138.

Finnerup, N.B., Haroutounian, S., Peter Kamerman et al. (2016). Neuropathic pain: an updated grading system for research and clinical practice. Pain, 157, 1599-606. 
Freeman, R., Baron, R., Bouhassira, D., Cabrera, J., \& Emir, B. (2014) Sensory profiles of patients with neuropathic pain based on the neuropathic pain symptoms and signs. Pain, 155, 367-76.

Gottrup H., Andersen J., Arendt-Nielsen L., Jensen T. (2000). Psychophysical examination in patients with post-mastectomy pain. Pain 87:275-84.

Guastella, V., Mick, G., \& Soriano, C. (2011). A prospective study of neuropathic pain induced by thoracotomy: incidence, clinical description, and diagnosis. Pain, 152, 74-81.

Hamdan, A., Luna, J.D., Del Pozo, E., \& Galvez, R. (2014). Diagnostic accuracy of two questionnaires for the detection of neuropathic pain in the Spanish population. European J Pain, 18, 101-109.

Høimyr, H., Rokkones, K. A., von Sperling, M. L., Finnerup, K., Jensen, T. S., \& Finnerup, N. B. (2011). Persistent pain after lymph node excision in patients with malignant melanoma is neuropathic. Pain, 152, 2721-2728.

Jensen, T.S., Baron, R., Haanpaa, M., Kalso, E., Loeser, J.D., Rice, A.S., \& Treede, R.D. (2011). A new definition of neuropathic pain. Pain, 152, 2204-2205.

Jensen, M.P., Chodroff, M.J., \& Dworkin, R.H. (2007). The impact of neuropathic pain on health-related quality of life: review and implications. Neurology, $68,1178-$ 1182.

Kaunisto, M.A., Jokela, R., Tallgren, M., Kambur, O., Tikkanen, E., Tasmuth, T., ... \& Kalso, E.A. (2013). Pain in 1,000 women treated for breast cancer: A prospective study of pain sensitivity and postoperative pain. Anesthesiology, 119, 1410-1421. 
Mathieson, S., Maher, C.G., Terwee, C.B., de Campos, T.F., \& Lin, C.W.C. (2015). Neuropathic pain screening questionnaires have limited measurement properties. A systematic review. J Clin Epidemiology, 68, 957-966.

Mustonen, L., Aho, T., Harno, H., Sipilä, R., Meretoja, T., \& Kalso, E. (2019). What makes surgical nerve injury painful? A 4- year to 9-year follow-up of patients with intercostobrachial nerve resection in women treated for breast cancer. Pain, 160, 246-256.

Pereira, S., Fontes, F., Sonin, T., Dias, T., Fragoso, M.,...\& Lunet, N. (2017). Neuropathic pain after breast cancer treatment: characterization and risk factors. $J$ Pain Symptom Manage, 54, 877-888.

Perez C., Galvez R., Huelbes S., Insausti J., Bouhassira D., Diaz S., Rejas J. (2007). Validity and reliability of the Spanish version of the DN4 (Douleur Neuropathique 4 questions) questionnaire for differential diagnosis of pain syndromes associated to a neuropathic or somatic component. Health and Quality of Life Outcomes, 5:66.

Sullivan, M.J.L., Bishop, S., \& Pivik, J. (1995). The pain catastrophizing scale: development and validation. Psychological Assessment, 7, 524-532.

Themistocleous A.C., Ramirez J.D., Shillo P.R., Lees J.G., Selvarajah D., Orengo D., ... \& Bennett D.L.H. (2016). The pain in neuropathy study (PiNS): a crosssectional observational study determining the somatosensory phenotype of painful and painless diabetic neuropathy. Pain, 157, 1132-45.

Timmerman, H., Steegers, M.A.H., Huygen, F.J.P.M., Goeman, J.J., van Dasselaar, N.T., Schenkels, M.J., ... \& Vissers, K.C.P. (2017). Investigating the validity of the DN4 in a consecutive population of patients with chronic pain. PLOS ONE, 12, e0187961. 
VanDenKerkhof, E.G., Stitt, L., Clark, A.J., Gordon, A., Lynch, M., Morley-Forster, P.K., ... \& Moulin, D.E. (2018). Sensitivity of the DN4 in screening for neuropathic pain syndromes. Clin J Pain, 34, 30-36.

Vilholm O.J., Cold, S., Rasmussen L. \& Sindrup S.H. (2009). Sensory function and pain in a population of patients treated for breast cancer. Acta Anaesthesiol Scand 53:800-6.

vonHehn C.A., Baron R. \& Woolf C.J. (2012). Deconstructing the neuropathic pain phenotype to reveal neural mechanisms. Neuron 73:638-52.

Woolf, C.J., \& Mannion, R.J. (1999). Neuropathic pain: aetiology, symptoms, mechanisms, and management. The Lancet, 353, 1959-1964.

\section{Figure legends}

\section{Figure 1.}

Patient flow chart.

\section{Figure 2.}

Sensory descriptors and sensory abnormalities according to the Douleur Neuropathique 4 (DN4) items. Patients with definite neuropathic pain (NP) reported more often electric shocks, tingling, pins and needles, and numbness at the neuroanatomically plausible area. Patients with definite NP showed most often hypoesthesia to touch and pinprick. For two-tailed significance: ${ }^{*} p<0.05,{ }^{* *} p<0.001$.

\section{Figure 3.}

The Receiver operating characteristic (ROC) curves for the interview part (items 1 to 7, DN4i, dashed line) and for the full version (items 1 to 10 , DN4, solid line) of the Douleur Neuropathique 4 questionnaire. 


\section{Table 1.}

Patient demographics and clinical features.

\begin{tabular}{|c|c|c|c|}
\hline & $\begin{array}{l}\text { Possible } \\
\text { NP }\end{array}$ & $\begin{array}{l}\text { Definite } \\
\text { NP }\end{array}$ & p-value \\
\hline Patients, $\mathrm{n}$ & 27 & 136 & \\
\hline Age, years, mean (SD) & $64.9(6.9)$ & $60.4(8.0)$ & $0.007^{\mathrm{a}}$ \\
\hline Time from index surgery, months, mean (SD) & $75.4(13.1)$ & $77.7(13.4)$ & $0.418^{\mathrm{a}}$ \\
\hline $\mathrm{BMI}, \mathrm{kg} / \mathrm{m}^{2}$, mean (SD) & $27.6(4.1)$ & $26.3(3.9)$ & $0.114^{\mathrm{a}}$ \\
\hline $\begin{array}{l}\text { Self-reported pain }{ }^{c} \text { NRS } \geq 4 \text { in the surgical area, } \\
\text { number (\%) }\end{array}$ & $5(18.5)$ & $30(22.1)$ & $0.497^{b}$ \\
\hline Evoked pain ${ }^{d}$ NRS $\geq 4$ in the surgical area, number $(\%)$ & $14(56.0)$ & $86(64.7)$ & $0.410^{b}$ \\
\hline $\begin{array}{l}\text { Self-reported }{ }^{c} \text { or evoked pain }{ }^{d} \text { NRS } \geq 4 \text { in the surgical } \\
\text { area, number }(\%)\end{array}$ & $17(63.0)$ & $96(70.6)$ & $0.433^{b}$ \\
\hline Pain elsewhere in the body ${ }^{\mathrm{e}} \mathrm{NRS} \geq 4$, number $(\%)$ & $6(23.1)$ & $53(44.5)$ & $0.044^{b}$ \\
\hline $\begin{array}{l}\text { Current use of TCA, gabapentinoids, or SNRI, number } \\
\text { (\%) }\end{array}$ & $2(7.4)$ & $7(5.1)$ & $0.639^{b}$ \\
\hline $\begin{array}{l}\text { Current use of NSAID, acetaminophen, or mild opioid, } \\
\text { number (\%) }\end{array}$ & $3(11.1)$ & $26(19.1)$ & $0.320^{b}$ \\
\hline \multicolumn{4}{|l|}{ Breast surgery type, number (\%) } \\
\hline BCS & $18(66.7)$ & $66(48.5)$ & \multirow[t]{2}{*}{$0.085^{b}$} \\
\hline Mastectomy & 9 (33.3) & $70(51.5)$ & \\
\hline \multicolumn{4}{|l|}{ Axillary surgery type, number (\%) } \\
\hline SLNB & $4(14.8)$ & $11(8.1)$ & \multirow[t]{2}{*}{$0.269^{k}$} \\
\hline ALND & $23(85.2)$ & $125(91.9)$ & \\
\hline \multicolumn{4}{|l|}{ Handling of ICBN, number (\%) } \\
\hline Partially resected & $22(81.5)$ & $88(64.7)$ & \multirow[t]{2}{*}{$0.089^{b}$} \\
\hline Totally resected & $5(18.5)$ & $48(35.3)$ & \\
\hline Chemotherapy, number (\%) & $20(74.1)$ & $121(89.0)$ & $0.039^{b}$ \\
\hline Radiotherapy, number (\%) & $22(81.5)$ & $104(76.5)$ & $0.570^{b}$ \\
\hline
\end{tabular}




\begin{tabular}{|c|c|c|c|}
\hline Endocrine therapy, number (\%) & $22(81.5)$ & $117(86.0)$ & $0.542^{b}$ \\
\hline & & & \\
\hline \multicolumn{4}{|c|}{$\begin{array}{l}\text { P-values < } 0.05 \text { are shown in bold. }{ }^{a} \text { Student's t-test. }{ }^{b} \mathrm{X}^{2} \text {-test. }{ }^{\mathrm{c}} \text { Assessed as the worst pain intensity } \\
\text { during past week reported in Brief Pain Inventory (BPI). }{ }^{\text {E}} \text { Evoked pain was assessed at clinical sensory } \\
\text { examination; intensity rating is missing from three definite NP patients and two possible NP patients. } \\
\text { e Answers missing from } 17 \text { definite NP patients and from one possible NP patient. Abbreviations: } \\
\text { ALND, axillary lymph node dissection; BCS, breast conserving surgery; BMI, body mass index; ICBN, } \\
\text { intercostobrachial nerve; NP, neuropathic pain; NSAID, non-steroidal anti-inflammatory drugs; SD, } \\
\text { standard deviation; SLNB, sentinel lymph node biopsy; SNRI, serotonin-norepinephrine reuptake } \\
\text { inhibitors; TCA, tricyclic antidepressant. }\end{array}$} \\
\hline
\end{tabular}

This article is protected by copyright. All rights reserved 


\section{Table 2.}

DN4i sum score correlations with demographics.

\begin{tabular}{|l|c|c|}
\hline & $\begin{array}{c}\text { DN4i } \\
\text { sum score } \\
\text { correlation }\end{array}$ & $\begin{array}{c}\text { P-value } \\
\text { (two tailed) }\end{array}$ \\
\hline Age (years) & -0.322 & $<\mathbf{0 . 0 0 1}$ \\
\hline Intensity of self-reported pain (NRS) & 0.185 & $\mathbf{0 . 0 1 8}$ \\
\hline Intensity of evoked pain (NRS) & 0.168 & $\mathbf{0 . 0 3 4}$ \\
\hline Intensity of worst pain at the operated area (NRS) & 0.179 & $\mathbf{0 . 0 2 2}$ \\
\hline Intensity of other chronic pain (NRS) & 0.261 & $\mathbf{0 . 0 0 2}$ \\
\hline Anxiety (HADS-A) & 0.179 & $\mathbf{0 . 0 2 3}$ \\
\hline Depression (HADS-D) & 0.245 & $\mathbf{0 . 0 0 2}$ \\
\hline Pain catastrophizing (PCS) & 0.213 & $\mathbf{0 . 0 0 9}$ \\
\hline \multicolumn{2}{|l}{} \\
\hline
\end{tabular}




\section{Table 3.}

Sensitivity, specificity, positive and negative predictive values, and the 95\% confidence intervals for the interview part (items 1 to 7 ) and full version (items 1 to 10) of the Douleur Neuropathique 4 (DN4) questionnaire with cut-off values 3 and 4 , respectively.

\begin{tabular}{|l|l|c|c|c|c|c|}
\hline & & $\begin{array}{c}\text { Cut-off } \\
\text { value }\end{array}$ & $\begin{array}{c}\text { Sensitivity } \\
(\%)(95 \% \mathrm{Cl})\end{array}$ & $\begin{array}{c}\text { Specificity } \\
(\%)(95 \% \mathrm{Cl})\end{array}$ & $\begin{array}{c}\text { Positive predictive value } \\
(\mathrm{PPV})(\%)(95 \% \mathrm{Cl})\end{array}$ & $\begin{array}{c}\text { Negative predictive value } \\
(\mathrm{NPV})(\%)(95 \% \mathrm{Cl})\end{array}$ \\
\hline \multirow{2}{*}{ All patients $(\mathrm{n}=163)$} & Interview part (DN4i, items 1 to 7) & 3 & $66.2(58-74)$ & $77.8(57-90)$ & $93.8(86-97)$ & $31.3(21-44)$ \\
\cline { 2 - 7 } & Full version (DN4, items 1 to 10) & 4 & $79.4(71-86)$ & $92.6(74-99)$ & $98.2(93-99)$ & $47.2(33-61)$ \\
\hline \multirow{2}{*}{$\begin{array}{l}\text { Patients with } \\
\text { NRS } \geq 4 / 10(\mathrm{n}=113)\end{array}$} & Interview part (DN4i, items 1 to 7) & 3 & $67.7(57-77)$ & $76.5(50-92)$ & $94.2(85-98)$ & $29.5(17-45)$ \\
\cline { 2 - 7 } & Full version (DN4, items 1 to 10) & 4 & $79.2(69-87)$ & $94.1(69-99)$ & $98.7(92-99)$ & $44.4(28-62)$ \\
\hline
\end{tabular}




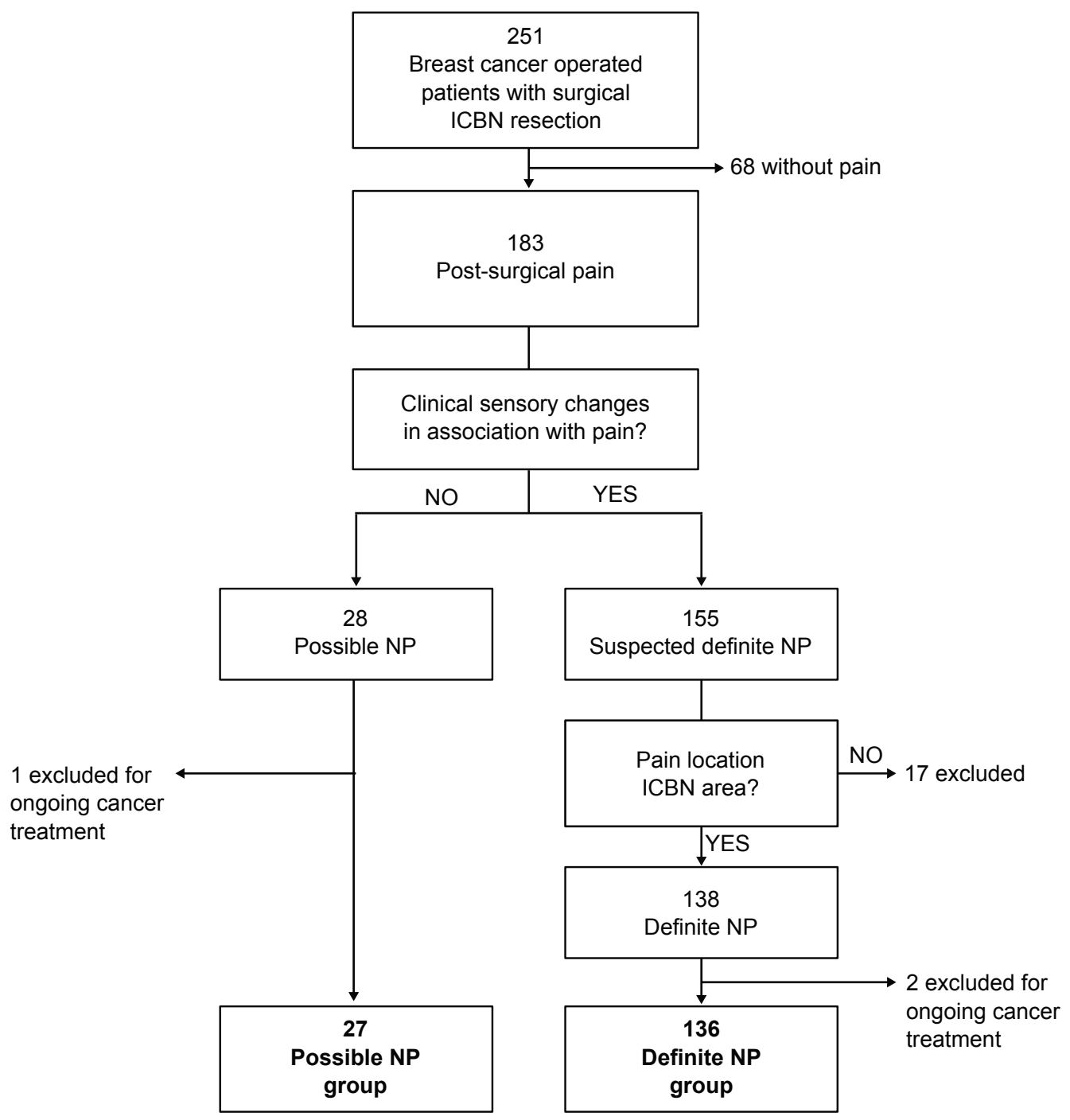




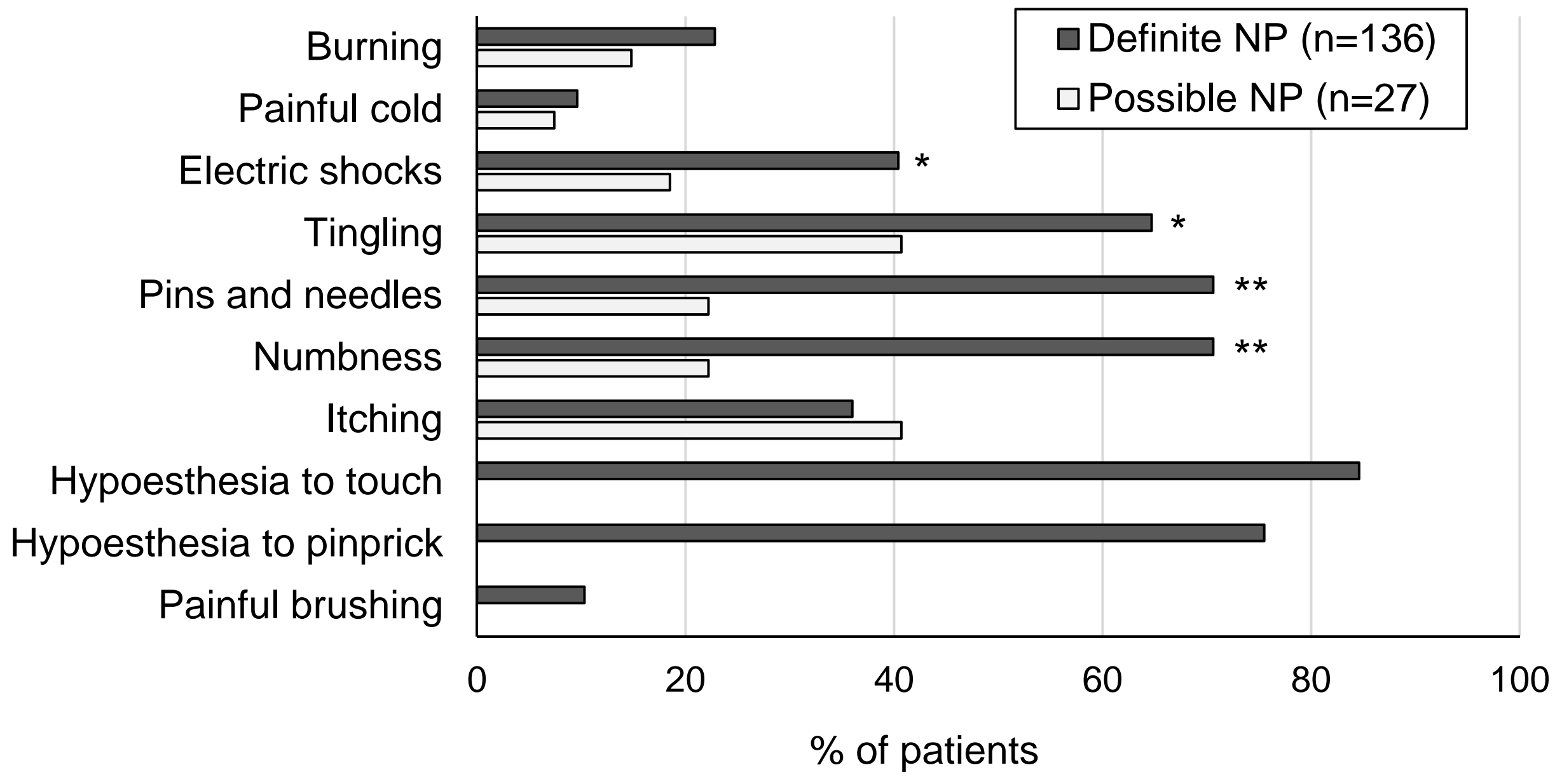




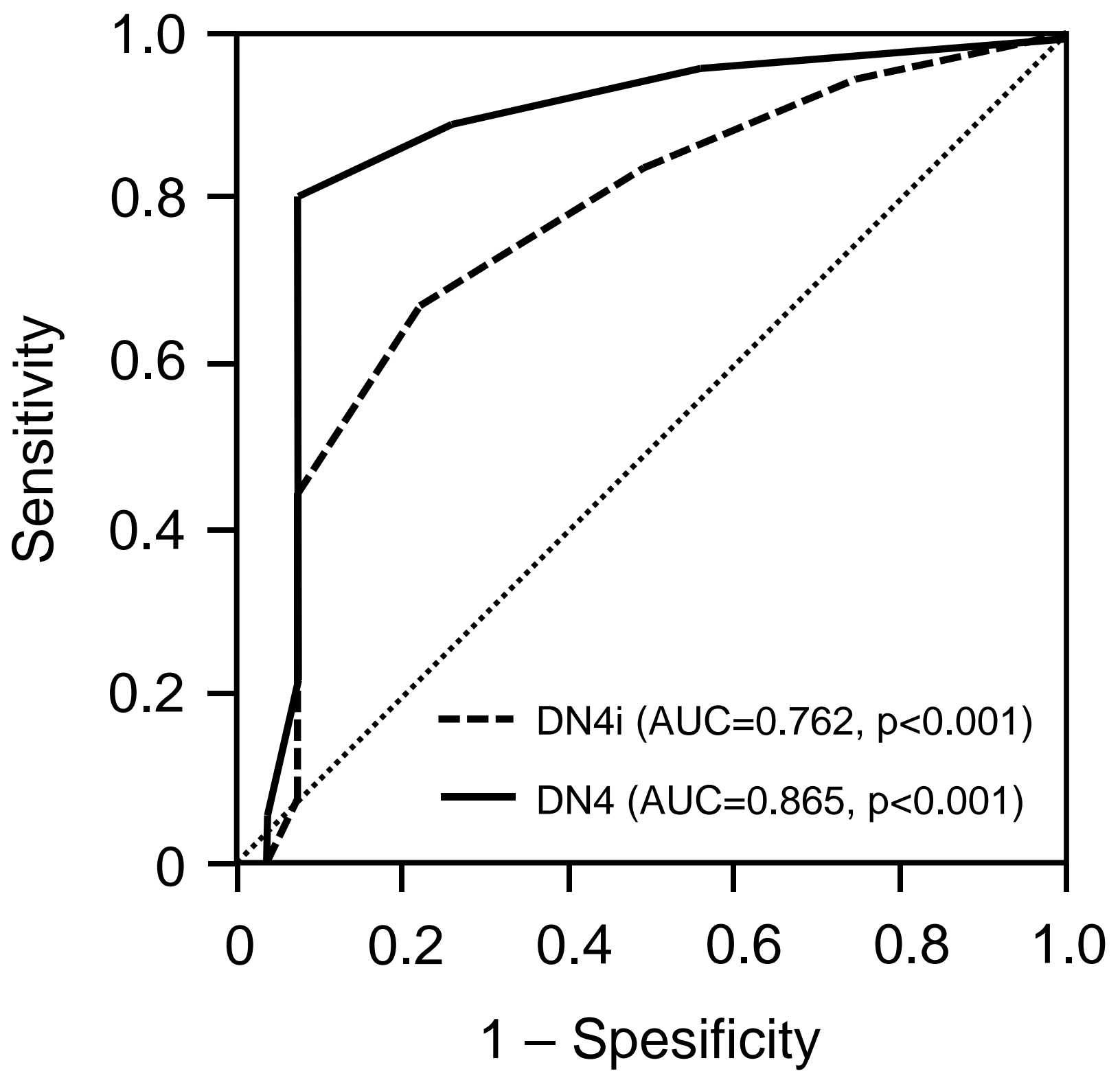

